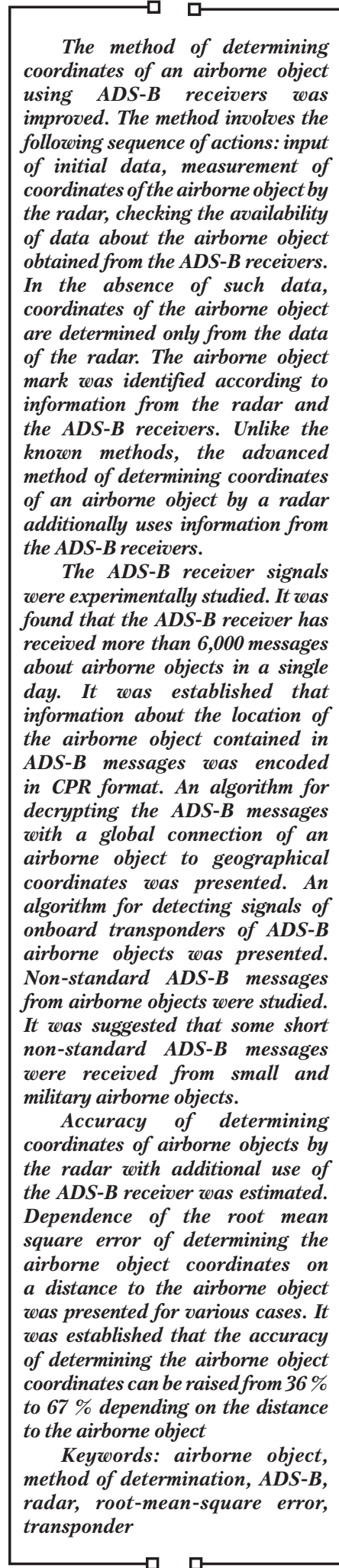

Received date 14.07.2021

Accepted date 18.08.2021

Published date 31.08.2021
UDC 621.396 .96

DOI: $10.15587 / 1729-4061.2021 .238407$

\title{
METHOD FOR DETERMINING COORDINATES OF AIRBORNE OBJECTS BY RADARS WITH ADDITIONAL USE OF ADS-B RECEIVERS
}

\section{Hennadi Khudov}

Corresponding author

Doctor of Technical Sciences, Professor, Head of Department Department of Radar Troops Tactic*

E-mail: 2345kh_hg@ukr.net

Oleksii Diakonov

$\mathrm{PhD}$, Associate Professor

Department of Programmable Electronics, Electrical Engineering and Telecommunications Admiral Makarov National University of Shipbuilding Heroiv Ukrainy ave., 9, Mykolayiv, Ukraine, 54025

$\mathrm{N}$ in a Kuchuk

Doctor of Technical Sciences, Associate Professor Department of Computer Engineering and Programming National Technical University "Kharkiv Polytechnic Institute"

Kyrpychova str., 2, Kharkiv, Ukraine, 61002

Volod y m y Maliuha

Doctor of Military Sciences, Senior Researcher, Head of Department Department of Anti-aircraft Missiles Tactic*

Kostiantyn Furmanov $\mathrm{PhD}$, Senior Researcher, Head of Department**

Ihor Mylashenko

$\mathrm{PhD}$, Senior Researcher Head of Department**

Yurii OIshevskyi

PhD, Senior Researcher, Head of Scientific Department The Scientific and Methodological Center of Scientific, Scientific and

Technical Activities Organization National Defence University of Ukraine named after Ivan Cherniakhovskyi Povitrofloski ave., 28, Kyiv, Ukraine, 03049

Stanislav Stetsiv Lecturer

Department of Missile Forces Hetman Petro Sahaidachnyi National Army Academy Heroiv Maidanu str., 32, Lviv, Ukraine, 79026

Yuriy Solomonenko $\mathrm{PhD}$, Deputy Head of Faculty of the Academic and Scientific Work Faculty of Radar-Technical Troops of Anti-Aircraft*

I ryna Yuzova $\mathrm{PhD}$, Lecturer

Department of Information Technologies Civil Aviation Institute

Klochkivska str., 228, Kharkiv, Ukraine, 61023 *Ivan Kozhedub Kharkiv National Air Force University Sumska str., $77 / 79$, Kharkiv, Ukraine, 61023

**The Scientific Research Center for Armed Forces Leadership Problems, Scientific Support of the Unified Automated Control System Armed Forces Central Scientific-Research Institute of Armed Forces of Ukraine Povitrofloski ave., 28, Kyiv, Ukraine, 03409

\section{Introduction}

Present-day armed conflicts feature the use of non-military methods to achieve goals. In hybrid wars of the new generation, the focus is shifted toward nonviolent actions. In so doing, ordinary, irregular, asymmetric efforts and undeclared military actions are applied. 
The peculiarity of conducting hybrid wars consists in that flights of civil aviation take place in normal mode. As a rule, two-coordinate surveillance radars with mechanical rotation in azimuth are used for conducting radar reconnaissance and airspace control (for example, P-18 type (Ukraine), P-18MA (Ukraine), P-18MU (Ukraine), P-18 Malakhit (Ukraine), etc. Such radars have low accuracy in determining coordinates of airborne objects (AO). As experience has shown, this can lead to the following:

- disguising airborne objects (mainly reconnaissance ones) as objects of civil aviation;

- difficulties in solving the problems of radar reconnaissance and airspace control by radar (radars);

- erroneous destruction of civil airborne objects, etc.

The use of known methods to improve the accuracy of determining coordinates of airborne objects leads to a growth of energy potential of an individual radar and a number of radars in common.

Therefore, it is important to improve the accuracy of determining coordinates of airborne objects in hybrid war conditions.

\section{Literature review and problem statement}

It is stated in [1] that the aggressor country has a possibility of alternative use of airborne objects violating state borders in conditions of present-day hybrid wars in the absence of open aggression. This is also confirmed in [2] on the example of hostilities in Iraq and Syria. Analysis [1,2] shows the possibility of disguising the airborne objects violating state borders as civilian scheduled airplanes.

It is noted in [3] that reconnaissance airborne objects will be violators of the state border most of all. This is stated only in [3]. No ways of detecting reconnaissance airborne objects are given in [3]. It is noted in [4] that contrary to international agreements, reconnaissance airborne objects can fly at a fairly short distance from civilian airplanes (about 300 m) (Fig. 1). As can be seen from Fig. 1, both civilian and reconnaissance airplanes (an airborne object violating the state border, e.g., a military airplane, pilotless vehicle, etc.) may be present in one resolution element. All this combined with the development and modernization of airborne objects, means of air attack, the growing intensity of air traffic in the radar inspection area complicates the tasks of radar reconnaissance and airspace control.

Range resolution in radar reconnaissance and airspace control is usually $600 \mathrm{~m}$ to $1800 \mathrm{~m}$ (e. g., [5]). In this case, radars will not be able to detect and track such reconnaissance airborne objects.

It is known that error in determining an arbitrary coordinate $R \sigma_{R}$ of the radar is determined from expression (1) [6]:

$$
\sigma_{R}=\frac{\Delta_{R}}{q}
$$

where $\Delta_{R}$ is $R$ coordinate resolution; $q$ is the signal-tonoise ratio.

Thus, taking into account expression (1) [6], the known methods of improving the accuracy of determining coordinates of the airborne object are aimed at improving the radar resolution and raising the signal-to-noise ratio.

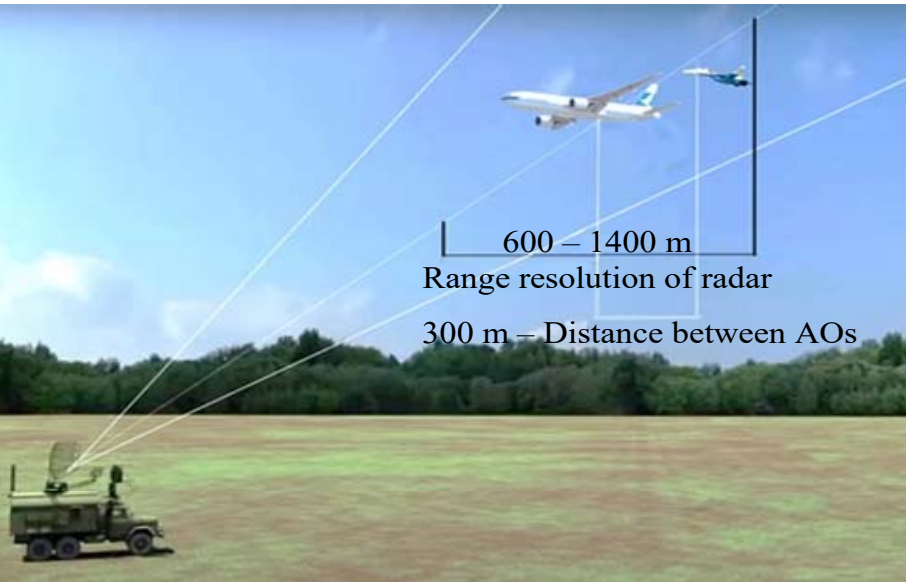

Fig. 1. Flight of a reconnaissance airborne object at a short distance from a civilian airplane

For example, the methods of spectral estimation are used in [7] to raise resolution. Such methods require additional use of a priori information about the signal parameters being estimated. It is almost impossible to obtain such a priori information in a case of detection of a state border violator. Therefore, the methods [7] are problematic to use. A method of raising the resolution of an unmanned aerial vehicle with optoelectronic surveillance equipment was proposed in [8]. The method [8] can be used to raise optical system resolution. To raise radio system resolution, method [8] is inapplicable. A method of super-resolution is offered in [9]. The main disadvantage of this study is the need to estimate the direct or inverse matrix of input signals. Application of the method of joint search and detection of objects is proposed in [10]. A disadvantage of this study consists in the need for two-stage decision-making procedures and significant time spent for making decisions. This requires a significant amount of calculations, formation of additive and multiplicative noise, reduction of the signal-to-noise ratio, etc.

Petrov-Galerkin's approach to improving the radar resolution and Hallström's discerning strategy of solving operator equations are used in the projection method [11]. This method requires additional a priori information (number, position, shape of individual elements of airborne object scattering) as well as knowledge of the type of basic functions to approximate the scatter surface. The disadvantage of this method includes inaccurate or insufficient a priori information, lack of a methodology of forming a priori information to solve practical problems and significant computational costs.

Thus, the main disadvantages of existing methods of improving the radar resolution are as follows:

- the need for a priori information to provide stability and satisfactory computational complexity;

- significant complication of processing algorithms and a sharp rise of computational costs with an increase in the number of discerning signals (number of airborne objects);

- the need for an appreciably small step in the calculation of the objective function when processing the radar signals;

- significant complication of processing algorithms when discerning a mixture of non-orthogonal signals, etc.

To raise the signal-to-noise ratio (reduction of the error in determining coordinates of airborne objects (expression (1)), the study [12] proposes the integration of surveillance radars in multi-radar networks. Methods of coherent signal processing 
in a multi radar net consisting of two surveillance radars were developed in [13]. Integration of radars in a multi-radar network $[12,13]$ leads to a greater number of surveillance radars and necessitates synchronous operation of surveillance radars in space and time.

To raise the signal-to-noise ratio, the following measures were proposed in [14-16]:

- compaction of disposal of surveillance radars [14];

- use of radars of different ranges [15];

- complication of radar information processing algorithms [16].

The main disadvantages of $[14-16]$ include the need to raise the energy potential of a particular radar, the number of radars, the complexity of methods of processing radar information, etc.

The use of phased arrays in radars as antenna systems is proposed in [17]. This will definitely raise the signal-tonoise ratio. However, restructuring of the antenna system is impossible when the radar is in position and radar reconnaissance and airspace control are conducted.

The use of an active system with external illumination by signals of cellular communication stations is proposed in [18]. A case of detection of airborne objects with the use of a two-position system with additional use of cellular communication signals is considered in [19]. The additional use of cellular communication signals has been shown to raise the signal-to-noise ratio and, consequently, the accuracy of determining airborne object coordinates. The main disadvantages of $[18,19]$ include the complexity of the synchronization of radars and cellular communication stations and the unresolved issue of optimizing the geometry of multi-position radar systems.

Methods of raising the signal-to-noise ratio through additional use of signals from space navigation systems are proposed in [20]. Synthesized algorithms of processing the navigation signals scattered by airborne objects and a structure of consumer's ground receiving equipment are considered.

A method of raising the signal-to-noise ratio through the introduction of a diverted reception channel in the radar was proposed in [21]. The method involves coordinated signal processing, quadratic detection, weight summation of detector outputs in each processing channel and in each element corresponding to the discrete volume and the corresponding Doppler's discrete frequency. Calculation of parameters of detection zones of surveillance radars with additional use of a channel of diverted reception is given in [22]. The main disadvantage of [21,22] consists in the need to make design changes in the radar, presence of a powerful penetrating signal in the channel of diverted reception.

To improve the quality of radar reconnaissance of airspace, the use of ADS-B information was proposed in [23]. The information capabilities of ADS-B, types of ADS-B receivers are considered, examples of data received in ASTERIX protocol (category 21) and in Advanced Virtual RISC (AVR) codes are given. This study states that the use of ADS-B data can provide additional information on airborne objects. However, it just states the possibility of obtaining additional ADS-B information, does not calculate errors in determining coordinates of airborne objects, etc.

Therefore, the use of information from ADS-B receivers as additional information during radar reconnaissance and airspace control can significantly raise the accuracy of de- termining coordinates of the radar airborne objects. So, the development of a method of determining coordinates of the radar's airborne objects with the additional use of ADS-B receivers is an urgent task.

\section{The aim and objectives of the study}

The study objective implies the improvement of a method for determining the position of airborne objects with additional use of ADS-B receivers. This will improve the accuracy of determining coordinates of airborne objects violating the state borders.

To achieve this objective, it was necessary to solve the following tasks:

- to determine main stages of the method of determining coordinates of airborne objects with additional use of ADS-B receivers;

- to study signals of ADS-B receivers;

- to estimate the accuracy of determining coordinates of airborne objects of the radar with additional use of ADS-B receivers.

\section{The study materials and methods}

The underwritten methods were used in the study.

When determining the main stages of the method of determining coordinates of radar airborne objects with additional use of ADS-B receivers, the following methods were used: methods of radar theory, methods of probability theory and mathematical statistics, methods of system analysis, methods of multiposition radiolocation, methods of differential calculus, methods of digital signal processing and mathematical apparatus of matrix theory.

When studying the ADS-B receiver signals, methods of radar theory, methods of probability theory and mathematical statistics, methods of mathematical modeling, methods of differential calculus, methods of digital signal processing, mathematical apparatus of matrix theory, methods of the statistical theory of detection and measurement of radar signals were used.

When estimating the accuracy of determining coordinates of radar airborne objects with additional use of ADS-B receivers, methods of radar theory, methods of probability theory and mathematical statistics, methods of mathematical modeling, methods of multi-position radiolocation, methods of digital signal processing, and mathematical apparatus of matrix theory were used.

Analytical and empirical methods of comparative study were used in the estimation of the proposed solutions.

The following limitations and assumptions were elaborated during the study:

- two-coordinate surveillance radars with mechanical rotation of P-18 type (Ukraine), P-18MA type (Ukraine), P-18MU type (Ukraine), P-18 Malakhit (Ukraine) were considered;

- radar radio receivers were of digital design;

- it was considered that there are no radio interferences;

- unimpeded signal reception from onboard ADS-B transponder was provided;

- RLT-SDR receiver was used as an ADS-B receiver: DVB-T+FM+DAB 820T2 \& SDR (antenna included). 
5. The results obtained in studying the development of a method for determining coordinates of airborne objects

5. 1. Main stages of improving the method of determining coordinates of airborne objects

To define the main stages of the method of determining coordinates of airborne objects with additional use of ADS-B receivers, a case of positioning the ADS-B receiver at the radar position was considered (Fig. 2).

\section{$\mathrm{AO} 1$}

(without ADS-B transponder)

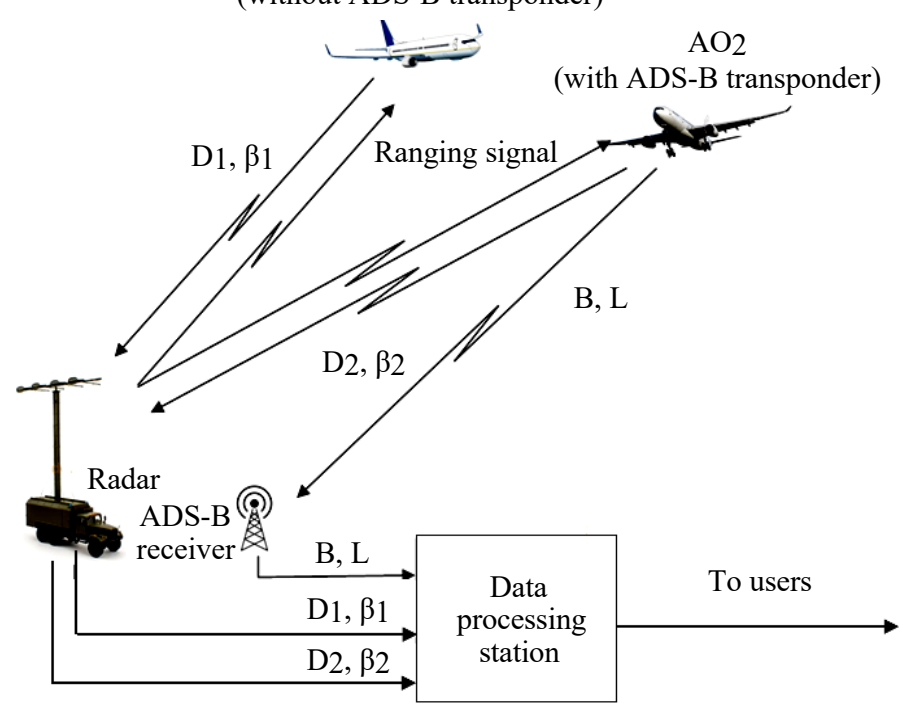

Fig. 2. Placement of the ADS-B receiver at the radar position

The radar measures angular coordinates $(D, \beta)$ where $D$ is the distance to the airborne object, $\beta$ is azimuth to the airborne object. In so doing, it is possible to find in the radar inspection area the airborne objects that are not equipped (airborne object 1 (AO1) and equipped (airborne object $2(\mathrm{AO} 2)$ ) with ADS-B transponders. In the case of equipping the AO2 with an ADS-B transponder, the ADS-B receiver will measure geodetic coordinates of the $\mathrm{AO} 2(B, L)$ where $B$ is latitude, $L$ is longitude.

The main stages of the algorithm of improving the method of determining coordinates of the airborne object with additional use of the ADS-B receivers are shown in Fig. 3.

In this case, the airborne object will be understood as AO2 equipped with an ADS-B transponder (Fig. 2).

The method of determining the $\mathrm{AO}$ coordinates using ADS-B receivers involves the following basic steps:

1. Input of source data: $\mathrm{BC}$ of the radar (resolution by the range and angular coordinates, accuracy of determining the $\mathrm{AO}$ coordinates, etc.), $\mathrm{BC}$ of $\mathrm{ADS}-\mathrm{B}$ receivers.

2. Measuring the AO coordinates.

3. Checking availability of $\mathrm{AO}$ data from ADS-B receivers.

4. In the absence of AO data from ADS-B receivers, they are determined using the radar data solely.

5. When data from ADS-B receivers are available, the $\mathrm{AO}$ coordinates are measured using the data from ADS-B receivers.

6. Identification of the mark from the airborne object according to the radar and ADS-B data (speed, course, coordinates, etc.). The airborne object height is taken into account only when using three-coordinate radars.
7. Determining the AO coordinates using data from ADS-B receivers (AO type, registration number, affiliation, call sign, coordinates, etc.).

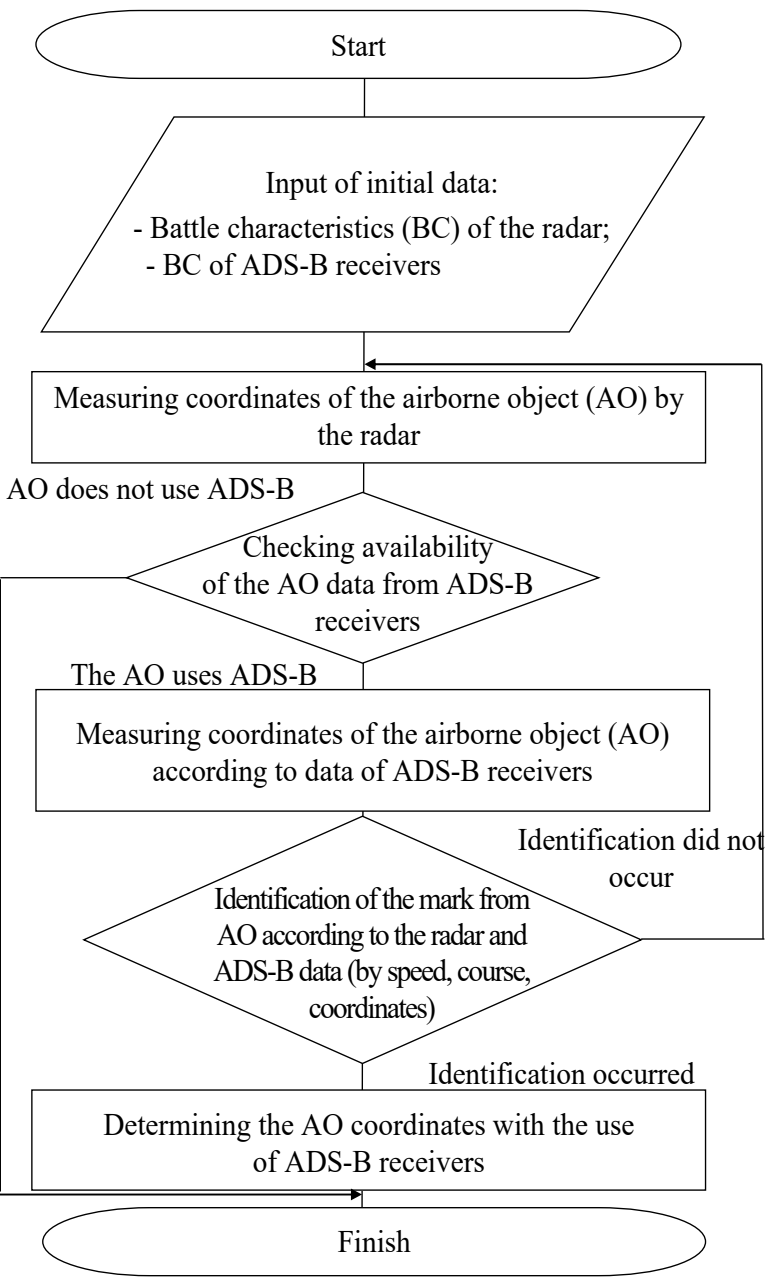

Fig. 3. Main stages of the algorithm of improving the method of determining the airborne object coordinates by a radar with additional use of ADS-B receivers

Thus, a method of determining coordinates of the radar airborne object has been developed in which (unlike the known methods) information of ADS-B receivers is additionally used.

\section{2. Experimental studies of the ADS-B receiver} signals

5. 2. 1. Initial data on conducting experimental studies

Experimental studies of the ADS-B receiver signals were conducted based on Admiral Makarov National University of Shipbuilding (Mykolaiv, Ukraine). The RLT-SDR receiver was used: DVB-T+FM+DAB 820T2 \& SDR (antenna included) (Fig. 4).

The receiver specification:

- frequency range: $24-1750 \mathrm{MHz}$;

- modulation type: FM, AM;

- bandwidth: 0.25-3 MHz;

- sensitivity: $220 \mathrm{mV}$;

- input resistance in reception: $50 \mathrm{Ohms}$;

- range filters: external only;

- bit-to-digital converter bit size: 8 bits; 
- dynamic range: $50 \mathrm{~dB}$;

- delay of signal detection: $340 \mathrm{~ms}$;

- interface: USB 2.0 .

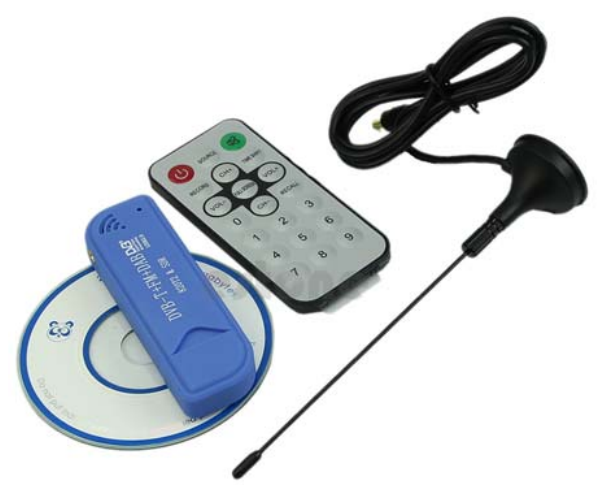

Fig. 4. RLT-SDR receiver: DVB-T+FM+DAB 820T2 \& SDR and a $15 \mathrm{~cm}$ antenna included

\section{2. 2. Experimental data obtained in one observa-} tion day

The ADS-B receiver (China) received more than 6,000 messages on airborne objects in one day. For example, airborne object 50816F (a civil Embraer airplane, Brazil) was observed for about 5 minutes and for about 10 minutes. Fig. 5, 6 show information from the Flightradar24 website (the site server in Sweden) regarding the airborne object 50816F (civil airplane Embraer). It was established that this airplane was flying on Kyiv-Kherson and Kherson-Kyiv routes.

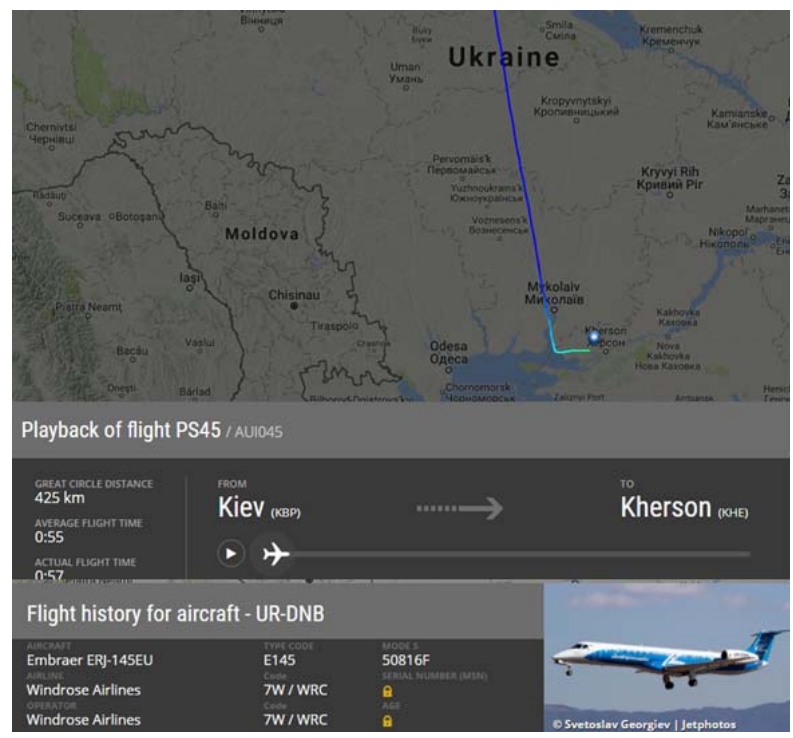

Fig. 5. Information from Flightradar24 website regarding the airborne object 50816F (Kyiv-Kherson flight)

More than 800 messages were received from the airborne object 4B906C (French Airbus A320 civil airplane). It was established that this civilian airplane was flying from Kharkiv to Istanbul (Fig. 7).

The solid blue line in Fig. 5-7 marks flights of airborne objects in the route map. ADS-B messages from airborne object transponders were decrypted.

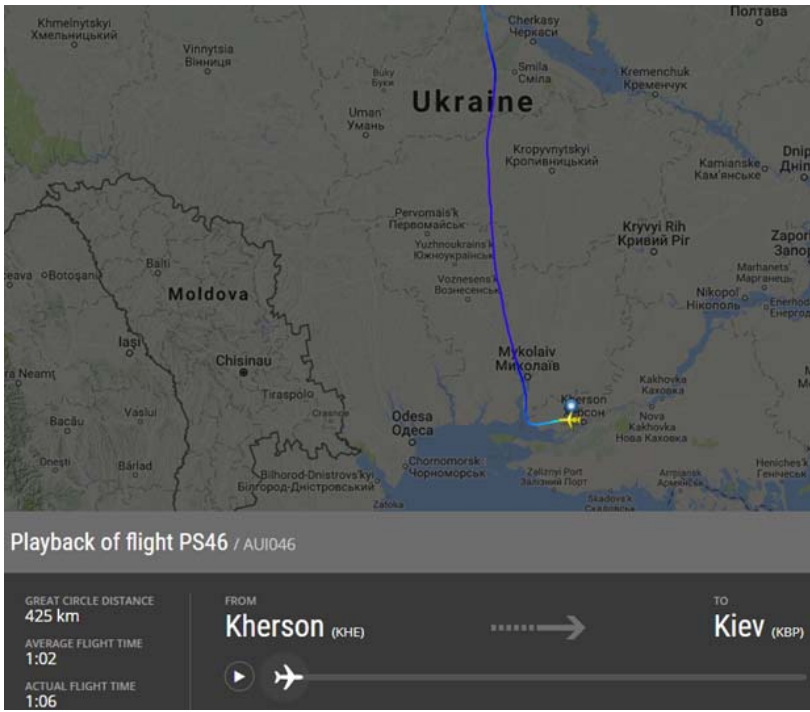

Fig. 6. Information from Flightradar24 website regarding airborne object 50816F (Kherson-Kyiv flight)

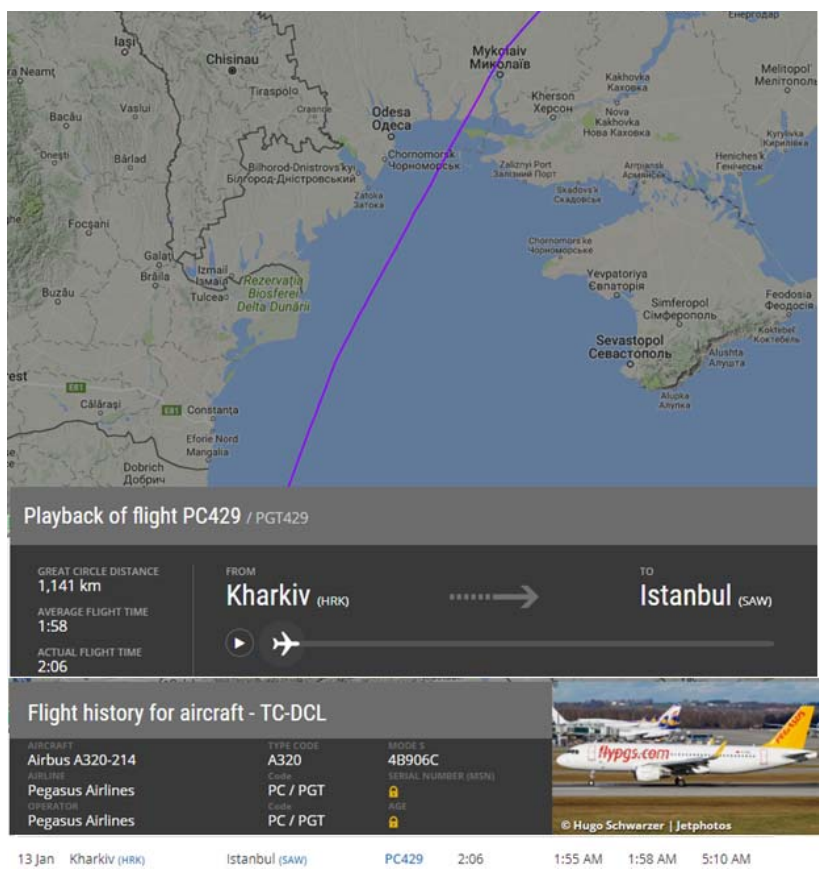

Fig. 7. Information from Flightradar24 website regarding airborne object 4B906C (Kharkiv-Istanbul flight)

\section{2. 3. Decrypt the ADS-B messages}

Information on airborne object location in the ADS-B messages is encoded in Compact Position Reporting (CPR) format aimed to encode more characters in geographic coordinates using fewer bits. The main idea of CPR consists in dividing the earth's surface into zones and transmitting the zone number instead of geographical coordinates. The number of latitude zones from the equator to the pole is $\mathrm{NZ}=15$ for Mode-S. The number of longitude zones NL depends on latitude (lat) and is given by expression (2).

The number of longitude zones varies from 1 for polar latitudes to 59 for equatorial latitudes. 


$$
\mathrm{NL}(\text { lat })=\text { floor }\left(\frac{2 \pi}{\arccos \left(1-\frac{1-\cos \left(\frac{\pi}{2 N Z}\right)}{\cos ^{2}\left(\frac{\pi}{180} l a t\right)}\right)}\right) \text {. }
$$

Two messages, multiple and non-multiple, are generated to initially link the airborne object to geographical coordinates. Knowing the previous location of the airborne object, information about geographical coordinates can be extracted from each message. When the airborne object is globally connected to geographical coordinates, the decryption algorithm is as follows:

1. The obtained data $L a t_{\mathrm{cprEven}}, L a t_{\mathrm{cprOdd}}, \operatorname{Lon}_{\mathrm{cprEven}}$, Loncprodd are transformed into the format with a floating point and normalized by $2^{17}$.

2. The latitude index is calculated from expression (3):

$$
j=\text { floor }\left(59 L a t_{\text {cprEven }}-60 L a t_{\text {cprOdd }}+0.5\right) \text {. }
$$

3. Relative latitude is calculated from expressions (4), (5):

$$
\begin{aligned}
& L a t_{\text {Even }}=\mathrm{d} L a t_{\text {Even }}\left(\bmod (j, 60)+L a t_{\text {cprEven }}\right) ; \\
& L a t_{\text {Odd }}=\mathrm{d} L a t_{\text {Odd }}\left(\bmod (j, 59)+L a t_{\text {cprOdd }}\right),
\end{aligned}
$$

where

$$
\mathrm{d} \operatorname{Lat}_{\mathrm{Even}}=(360 /(4 \mathrm{NZ}))=(360 / 60) ;
$$

$\mathrm{d}$ Lat $_{\mathrm{Odd}}=(360 /(4 \mathrm{NZ}-1))=(360 / 59)$ are constants.

4. To ensure membership of the range $\left[-90^{\circ}, 90^{\circ}\right]$, the calculated values are corrected, if necessary, according to expressions (6), (7):

$$
\begin{aligned}
& L a t_{\text {Even }}=L a t_{\text {Even }}-360 \text { if }\left(L a t_{\text {Even }} \geq 270\right) \\
& L a t_{\text {Odd }}=L a t_{\text {Odd }}-360 \text { if }\left(L a t_{\text {Odd }} \geq 270\right) .
\end{aligned}
$$

5. Later messages are used as the final value of latitude (expression (8)):

$$
L a t=\left\{\begin{array}{c}
L a t_{\text {Even }}, \text { if }\left(T_{\text {Even }} \geq T_{\text {Odd }}\right) ; \\
L a t_{\text {Odd }}, \text { otherwise. }
\end{array}\right.
$$

6. Longitude zone numbers are determined for both latitude values $\mathrm{NL}\left(\right.$ Lat $\left._{\text {Even }}\right)$ and $\mathrm{NL}\left(\right.$ Lat $\left._{\mathrm{Odd}}\right)$ using expression (2). If they are different, it is impossible to calculate global longitude and it is necessary to wait for the next pair of messages.

7. In the case of the coincidence of numbers of longitude zones for both values of latitude, it is necessary to calculate global longitude. If the time of multiple messages is greater than the aliquant time, the following calculations must be performed (expressions (9) to (12):

$$
n i=\max \left(\mathrm{NL}\left(\text { Lat }_{\mathrm{Even}}\right), 1\right) \text {; }
$$

$$
\mathrm{d} L o n=360 / n i \text {; }
$$

$$
\begin{aligned}
& m=\text { floor }\left(\text { Lon }_{\text {cprEven }}\left[\mathrm{NL}\left(\text { Lat }_{\text {Even }}\right)-1\right]-\right. \\
& \left.- \text { Lon }_{\text {cprOdd }} \mathrm{NL}\left(\text { Lat }_{\text {Even }}\right)+0.5\right) \text {; } \\
& \text { Lon }=\mathrm{d} \operatorname{Lon}\left(\bmod (m, n i)+\text { Lon }_{\text {cprEven }}\right) .
\end{aligned}
$$

If otherwise, then expressions (13)-(16) are used:

$$
\begin{aligned}
& n i=\max \left(\mathrm{NL}\left(\text { Lat }_{\mathrm{Odd}}-1\right), 1\right) ; \\
& \mathrm{d} \text { Lon }=360 / n i ; \\
& m=\text { floor }\left(\text { Lon }_{\text {cprEven }}\left[\mathrm{NL}\left(\text { Lat }_{\text {Odd }}\right)-1\right]-\right. \\
& \left.- \text { Lon }_{\text {cprOdd }} \mathrm{NL}\left(\text { Lat }_{\text {Odd }}\right)+0.5\right) ; \\
& \text { Lon }=\mathrm{d} \text { Lon }\left(\bmod (m, n i)+\text { Lon }_{\text {cprOdd }}\right) .
\end{aligned}
$$

8. The longitude values exceeding $180^{\circ}$ are adjusted using expression (17):

$$
\text { Lon=Lon-360, if (Lon } \geq 180) \text {. }
$$

Information on the airborne object altitude above sea level comes in the same message as the geographical coordinates. Altitude calculations using it do not depend on the message parity and are always given in feet. The incoming data contain information on the accuracy of measuring the airborne object altitude. This information is given by the so-called Q-bit. If its value is " 0 ", then altitude discreteness discr is " 100 ", otherwise " 25 ". The Q-bit must be removed and the resulting value of $N$ converted into feet using expression (18):

$$
A l t=N^{*} \text { diskr-1000 (feet). }
$$

According to (18) and number of the bits bearing the altitude information (11 bits), the range of altitudes that can be encoded is 1000 to $2^{11-1} \times 25-1000=50,175$ feet or 305 to 15,293 meters for Q-bit=1.

Local connection of an airborne object to geographical coordinates presupposes knowledge of the reference point in the vicinity of which the airborne object is located. This can be data obtained from previous messages or data on the location of the receiving antenna. A message with any parity can be used. With local connection, latitude is calculated using expressions (19) to (21):

$$
L a t=\mathrm{d} L a t\left(j+L a t_{\mathrm{cpr}}\right) \text {; }
$$

$$
\mathrm{d} L a t=\left\{\begin{array}{l}
\frac{360}{4 N Z}=\frac{360}{60}, \text { if number of message is Even; } \\
\frac{360}{4 N Z-1}=\frac{360}{59}, \text { if number of message is Odd; }
\end{array}\right.
$$

$$
\begin{aligned}
& j=\text { floor }\left(\frac{L a t_{r e f}}{\mathrm{~d} L a t}\right)+ \\
& + \text { floor }\left(\frac{\bmod \left(L_{a t}, \mathrm{~d} L a t\right)}{\mathrm{d} L a t}-L a t_{c p r}+0.5\right),
\end{aligned}
$$

where $L a t_{r e f}$ is the width of the reference point.

With local connection, longitude is calculated using expressions (22) to (24): 
Lon $=\mathrm{d} \operatorname{Lon}\left(m+\operatorname{Lon}_{\mathrm{cpr}}\right)$;

$$
\begin{aligned}
& \mathrm{d} \text { Lon }=\left\{\begin{array}{l}
\frac{360}{\mathrm{NL}(\text { Lat })}, \text { if } \mathrm{NL}(\text { Lat })>0 ; \\
360, \text { if } \mathrm{NL}(\text { Lat })=0 ;
\end{array}\right. \\
& m=\text { floor }\left(\frac{\text { Lon }_{r e f}}{\mathrm{~d} L o n}\right)+ \\
& + \text { floor }\left(\frac{\bmod \left(\text { Lon }_{r e f}, \mathrm{~d} \text { Lon }\right)}{\mathrm{d} L o n}-\text { Lon }_{c p r}+0.5\right),
\end{aligned}
$$

where Lon $_{\text {ref }}$ is the longitude of the reference point.

\section{2.4. Detecting signals of onboard airborne tran-} sponders ADS-B

Information from the receiver output is immediately passed through a square-law detector (complex readings are replaced by amplitudes). Amplitudes at the output of the square-law detector are interpolated to raise quantization frequency from $2.4 \mathrm{MHz}$ to $12 \mathrm{MHz}$. Additional readings are built based on the reconstruction or anti-imaging FIR filter.

Detection of on-board signals begins with the selection of samples that exceed average background by $14 \mathrm{~dB}$, i.e. average level of amplitude throughout the frame is calculated and samples with amplitude not less than 5 times the average value are selected. For such samples, the zone of the possible presence of a signal with an indentation by samplesBeforeSyncPulse $=60$ samples before the selected sample and by samplesAfterMessage $=120$ samples after the expected long message is formed. The number of samples in the extended zone is 1524 (sync pulse length of 96 samples in the short message and 1248 samples in the long message). A standard sample correlation coefficient with a known sinc pulse is calculated for the first 85 samples of this zone. A sync pulse of a standard ADS-B message is shown in Fig. 8.

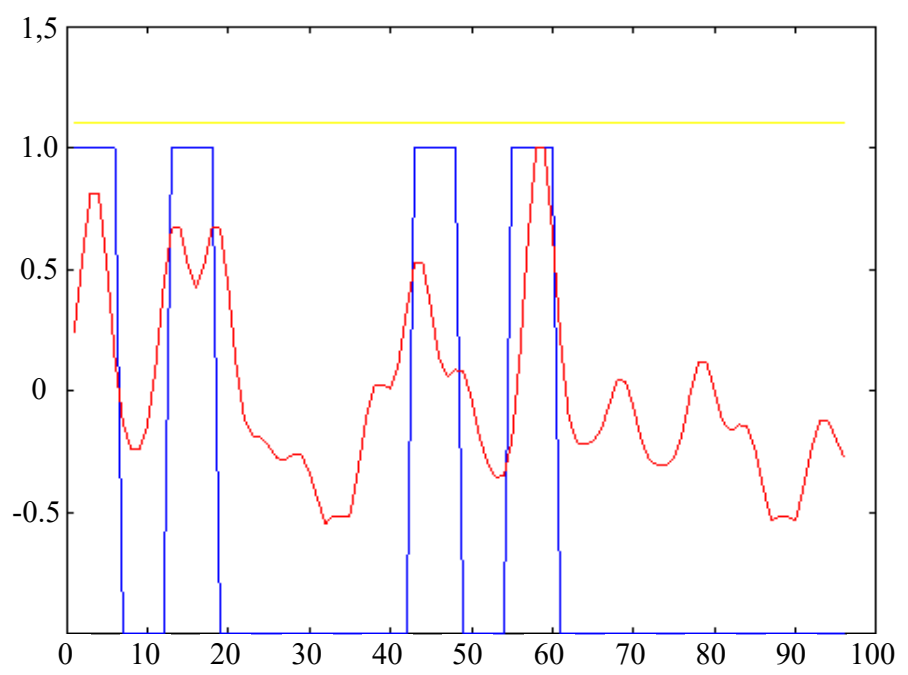

Fig. 8. Standard sync pulse of the ADS-B message

The received sync pulses are quite distorted (usually, the correlation coefficient does not exceed 0.7). In the algorithm under consideration, if the maximum coefficient among the calculated correlation coefficients exceeds the set threshold
corrThreshold $=0.68$, then this sample is taken as the beginning of the clock pulse and bits are allocated on the interval of the long message. " 1 " is formed if the sum of the first 6 samples exceeds the sum of the next 6 samples, otherwise " 0 " is formed. Regardless of the length of the transmitted message, it is decoded as a long one. The message is translated into hexadecimal form and the CRC code is checked. If the CRC matches, receiving a long message is guaranteed. If not, the received characters are truncated to 56 bits (14 digits) and the hypothesis of receiving a short message by a new calculation of the CRC code is checked. The crc $24 \mathrm{f}$ () function which implements a 24-bit CRC based on the universal comm.CRCDetector () function is used to check the CRC code. It should be noted that regardless of the length of the message, the probability of a random match of the 24-bit CRC code is equal to $\frac{1}{2^{24}} \approx 6 \times 10^{-8}$, which virtually guarantees error-free decoding of all messages.

\section{2.5. Study of non-standard ADS-B messages from} airborne objects

Non-standard ADS-B signals were detected during experimental studies. The CRC code matches such messages. However, such messages are not detected with the enabled detection of a given sync pulse or other systems for working with ADS-B Mode-S signals. Study of the Frame20210224.bin file with amplitude recordings during one day was performed by the ADS-B signal detection program with sync pulse detection on and off. It was established that with the sync pulse detection turned off, some standard signals are detected which are determined with the sync pulse detection on. Their AirplanesID board number was determined without errors but the rest of the message was received incorrectly, with substantial errors. In addition to the standard signals when detection of a given sync pulse is turned off, there was a significant number of non-standard short messages (or fragments of messages) starting with the letters VA and BE. For example, there were 13 detected standard signals for 13 detected standard signals. In total, the Frame20210224.bin file contained 239 short non-standard messages. The probable sync pulse of non-standard ADS-B messages is shown in Fig. 9.

The number of non-standard messages on airborne objects according to the results of analysis of the Frame20210224.bin file (a fragment) is given in Table 1. Examples of the results of searching for information on airborne objects on the Flightradar24 website according to board numbers given in Table 1 are shown in Fig. $10-12$.

According to the analysis results, a substantial part of board numbers of airborne objects received in short non-standard ADS-B messages is in the Flightradar24 net. However, there is almost no information about types of airborne objects or their flights, except for some boards. For example, there is no information on the following airborne objects with flight numbers A106CD, 975327, 975328, 975A60, 975333, 972D15, 9720C2, 977082, 97708D, 9720C2 in the Flightradar24 network. The specialized aviation online register Planespotters.net does not contain any information about airborne objects with determined board numbers. 


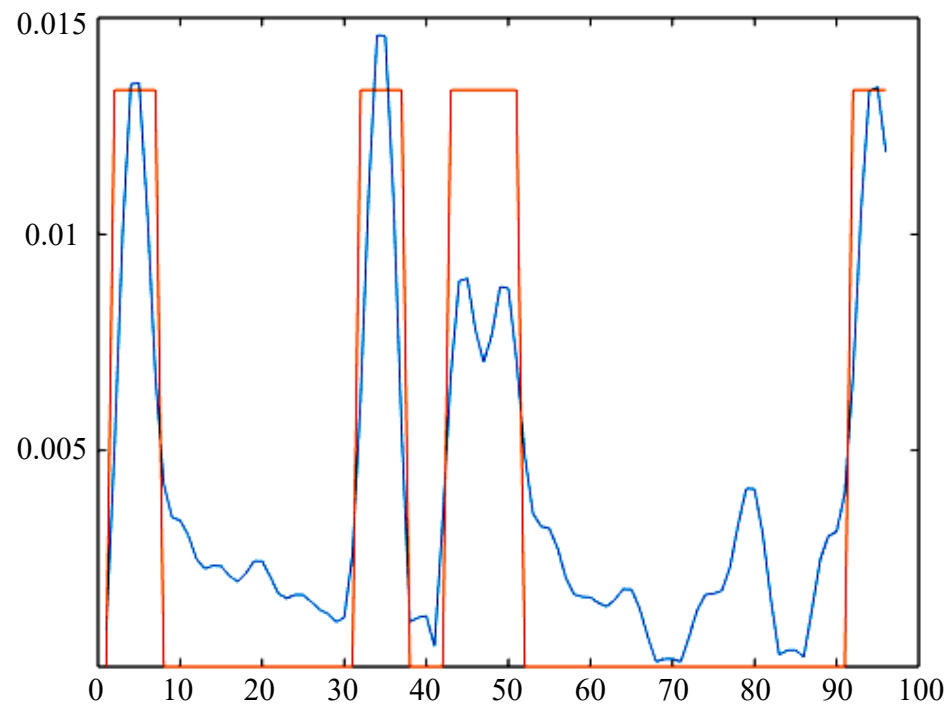

Fig. 9. Probable sync pulse of non-standard ADS-B messages
Attention was drawn to some board numbers of airborne objects from which non-standard ADS-B messages were received (e.g. A106CD). They were compared with the ICAO code format of the airborne object. Based on this information, it can be assumed that the first part of the board number of airborne objects may be an ICAO code. For example, A106CD would correspond to ICAO code A106. This assumption is relevant for small airplanes. However, in this case, 256 values remain for the individual number in the series which is quite small. A complete list of ICAO codes is not freely available, so it is impossible to confirm or deny this assumption.

Thus, it can be assumed that some short non-standard ADS-B messages are received from small airplanes. The lack of any data on the rest of the detected messages suggests that military airborne objects are their source.

Table 1

The number of non-standard messages on airborne objects according to the results of analysis of the Frame 20210224.bin file (fragment)

\begin{tabular}{|c|c|}
\hline Board number & Number of non-standard messages \\
\hline A10495 & 44 \\
\hline A1031A & 62 \\
\hline $972 \mathrm{D} 15$ & 7 \\
\hline $9720 \mathrm{C} 2$ & 13 \\
\hline A10495 & 44 \\
\hline $970 \mathrm{D} 04$ & 10 \\
\hline A1049E & 2 \\
\hline A103F6 & 1 \\
\hline A100F6 & 5 \\
\hline A105D1 & 11 \\
\hline A104CE & 11 \\
\hline 977082 & 20 \\
\hline $97708 \mathrm{D}$ & 17 \\
\hline A10596 & 7 \\
\hline A105D5 & 1 \\
\hline Total: & 255 \\
\hline
\end{tabular}

\section{Flight history for aircraft - N165DJ}

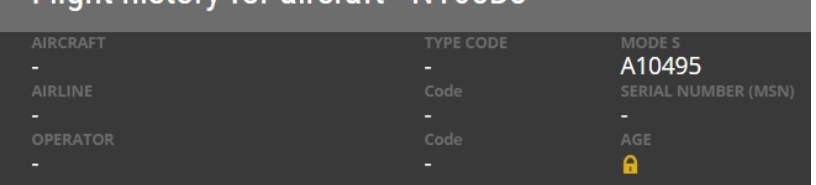

Fig. 10. Information taken from the Flightradar24 website on the airborne object with flight number A 10495

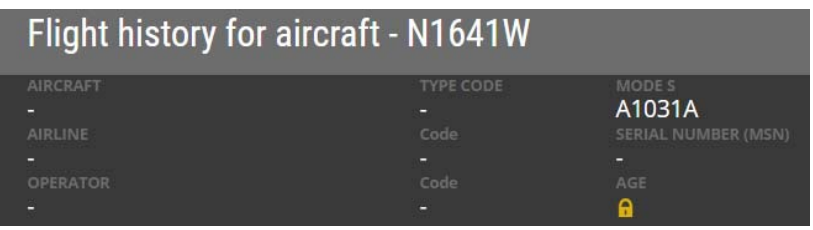

Fig. 11. Information taken from the Flightradar24 website on the airborne object with the flight number A1031A

\section{Flight history for aircraft - N164EH}

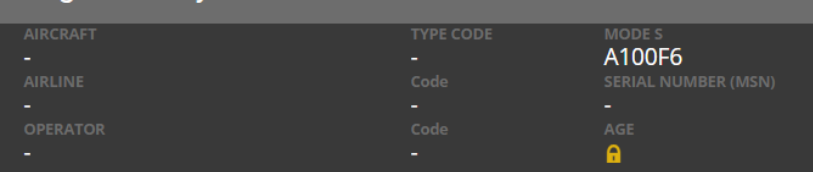

Fig. 12. Information taken from the Flightradar24 website on the airborne object with the flight number A100F6

5. 3. Estimation of accuracy of determining coordinates of airborne objects by a radar with additional use of the ADS-B receiver

Experimental studies of the method of determining coordinates of airborne objects with the additional use of the ADS-B receiver were conducted. P-18MA radars (Ukraine) were used. Let us make a comparative estimation of the accuracy of determining coordinates of airborne objects using various methods. Theoretical calculations were performed to raise the accuracy of determining coordinates of airborne objects. Root mean square (RMS) error of determining coordinates of the airborne object was used as an accuracy indicator (e.g., $[24,25])$. The resulting RMS error of determining coordinates of the radar airborne object with additional use of the ADS-B receiver is determined by expression (25):

$$
\sigma_{\Sigma}=\sqrt{\frac{\sigma_{\text {Radar }}^{2} \cdot \sigma_{\text {ADS-B }}^{2}}{\sigma_{\text {Radar }}^{2}+\sigma_{\text {ADS-B }}^{2}}},
$$

where $\sigma_{\Sigma}$ is the resulting RMS error of determining coordinates of the radar airborne object with additional use of the ADS-B receiver;

$\sigma_{\text {Radar }}$ is RMS error of determining coordinates of the airborne object using the radar solely;

$\sigma_{\mathrm{ADS}-\mathrm{B}}$ is the RMS error of determining coordinates of the airborne object using the ADS-B receiver.

Fig. 13 shows the dependence of RMS error of determining coordinates of the airborne object on the distance to the airborne object for various cases. For example, line 1 shows the dependence of $\sigma_{\text {Radar }}$ on the distance to the airborne object using the radar data only. Line 2 shows the dependence 
of $\sigma_{\mathrm{ADS}-\mathrm{B}}$ on the distance to the airborne object using ADS-B receivers. Line 3 shows the dependence of $\sigma_{\Sigma}$ on the distance to the airborne object using the radar data with additional use of the ADS-B receiver.

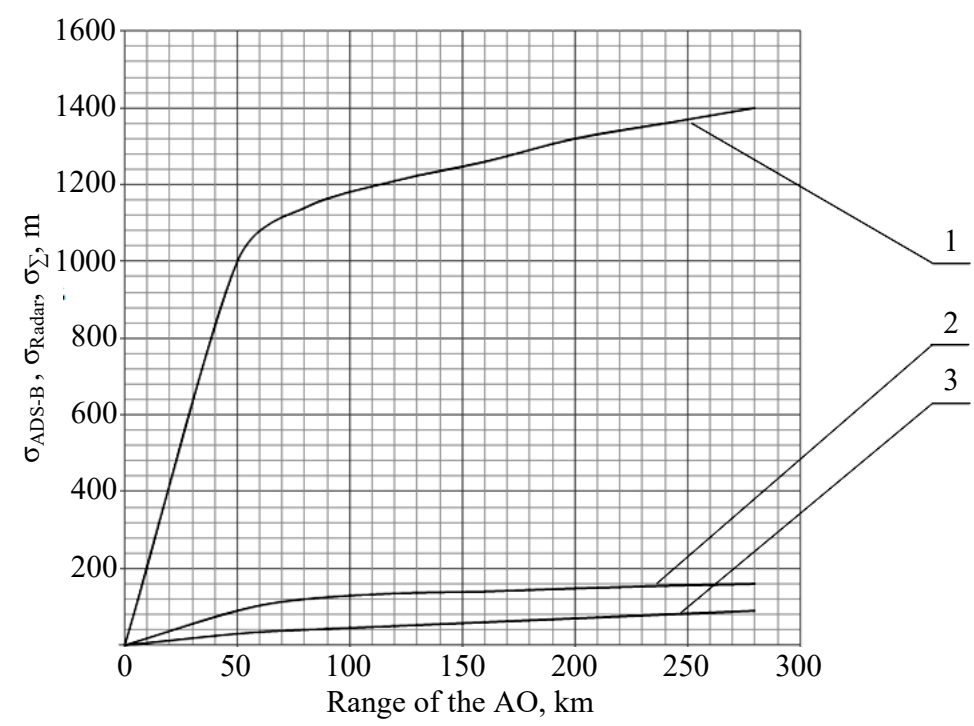

Fig. 13. Dependence of the root mean square error of determining coordinates of an airborne object on the distance to the airborne object for various cases

It can be concluded from the analysis of Fig. 13 that the accuracy of determining coordinates of the airborne object can be raised from $36 \%$ to $67 \%$ depending on the distance to it.

\section{Discussion of the results obtained in studying the development of a method of determining the airborne object coordinates}

The main stages of improvement of the method of determining coordinates of the radar airborne object with additional use of ADS-B receivers are given in Fig. 3. This method involves the following sequence of steps: input of initial data, measurement of coordinates of the airborne object using the radar, checking the availability of data about the airborne object obtained from the ADS-B receivers. In the absence of such data, coordinates of the airborne object are determined only by using the radar data. The mark from the airborne object is identified according to the radar and the ADS-B data. Thus, the method of determining coordinates of the radar airborne object has been improved. Information of ADS-B receivers is additionally used in this method, unlike the known methods.

Experimental studies of the ADS-B receiver signals were performed. The RLT-SDR receiver was used: DVB-T+ $+\mathrm{FM}+\mathrm{DAB} 820 \mathrm{~T} 2$ \& SDR (antenna included) (Fig. 4). It was found that more than 6,000 messages on airborne objects were received in one day of the ADS-B receiver operation. It was also found that more than 800 messages were received from $4 \mathrm{~B} 906 \mathrm{C}$ airborne object. It was established that it was a civilian airplane flying from Kharkiv to Istanbul (Fig. 7). The decryption of ADS-B messages is given. It was established that information about the location of the airborne object in ADS-B messages is encoded in $\mathrm{CPR}$ format. The main CPR idea consists in dividing the earth's surface into zones and transmitting the zone number instead of its geographical coordinates (2). An algorithm of decryption of the ADS-B messages with a global connection of airborne objects to geographical coordinates ((3)-(17)) was presented. An algorithm for detecting signals of onboard ADS-B transponders of airborne objects was given. A sync pulse of the standard ADS-B message was presented (Fig. 8). Non-standard ADS-B messages from airborne objects were studied. The probable sync pulse of non-standard ADS-B messages was presented (Fig. 9).

It has been suggested that some short non-standard ADS-B messages are received from small airplanes. Also, the lack of any data on the rest of the detected messages suggests that military airborne objects are their source.

Accuracy of determining coordinates of airborne objects by the radar with additional use of the ADS-B receiver was estimated. The P-18MA radar (Ukraine) was used. Value of RMS error was used as an indicator of accuracy (expression (25)). Dependence of the RMS error of determining the airborne object coordinates on the distance to the airborne object was shown for various cases (Fig. 13). It was established that the accuracy of determining the airborne object coordinates can be raised from $36 \%$ to $67 \%$ depending on the distance to the airborne object.

The study has the following limitations and assumptions: - surveillance two-coordinate radars with mechanical rotation of P-18 type (Ukraine), P-18MA (Ukraine), P-18MU (Ukraine), P-18 Malakhit (Ukraine) were considered;

- radar radio receivers are of digital design;

- it is assumed that there are no interferences;

- unimpeded signal reception from the onboard transponder ADS-B is provided;

- RLT-SDR receiver is used as the ADS-B receiver: DVB-T+FM+DAB 820T2 \& SDR (antenna included).

It is expedient that further studies should be focused on the development of methods for determining coordinates of airborne objects that are not equipped with ADS-B transponders and radars.

\section{Conclusions}

1. The advanced method of determining coordinates of airborne objects using the ADS-B receivers involves the following sequence of actions: input of initial data, measurement of coordinates of the airborne object by the radar, checking the availability of data received from ADS-B receivers about the airborne object. In absence of such data, coordinates of the airborne objects are determined only by using data of the radar. The airborne object mark is identified according to the data of the radar and the ADS-B receivers. Unlike the known methods, the developed method of determining coordinates of the airborne object additionally uses information of the ADS-B receivers.

2. Experimental studies of the ADS-B receiver signals were performed. It has been established that more than 6,000 messages on airborne objects were received in one day of the ADS-B receiver operation. It was established 
that information about the location of airborne objects in ADS-B messages is encoded in CPR format. An algorithm of decoding the ADS-B messages when the global connection of an airborne object to geographical coordinates was provided. An algorithm for detecting signals of onboard ADS-B transponders of airborne objects was presented. A study has been conducted on non-standard ADS-B messages from airborne objects. It has been suggested that some short non-standard ADS-B messages are received from small airplanes. The lack of any data on the rest of the detected messages suggests that military air objects are their source.

3. Accuracy of determining coordinates of airborne objects by the radar with additional use of the ADS-B receiver was estimated. Dependence of RMS errors of determining coordinates of airborne objects on the distance to the airborne object was presented for various cases. It was established that the accuracy of determining coordinates of the airborne object can be raised from $36 \%$ to $67 \%$ depending on the distance to the airborne object.

\section{References}

1. Deep, A. (2015). Hybrid War: Old Concept, New Techniques. Small Wars Journal. Available at: https://smallwarsjournal.com/jrnl/ art/hybrid-war-old-concept-new-techniques

2. Marton, P. (2017). Evolution in military affairs in the battlespace of Syria and Iraq. Corvinus Journal of International Affairs, 2 (2-3), 30-41. doi: https://doi.org/10.14267/cojourn.2017v2n2a3

3. Eurocontrol warns airlines of 'possible military action' in Syria. Available at: https://www.politico.eu/article/eurocontrol-warnsairlines-of-possible-military-action-in-syria-chemical-weapons/

4. Ministry of infrastructure of Ukraine. Ukrainian State Air Traffic Services Enterprise. Safety. Efficiency. Responsibility. Available at: https://uksatse.ua/index.php?lang=en

5. Ground-based long-range VHF band surveillance radar P-18MA (P-180U). Available at: https://www.aerotechnica.ua/en/p-18maen.html

6. Richards, M. A., Scheer, J. A., Holm, W. A. (Eds.) (2010). Principles of Modern Radar: Basic principles. IET. doi: https:// doi.org/10.1049/sbra021e

7. Marpl-ml, S. L. (1990). Tsifrovoy spektral'niy analiz i ego prilozheniya. Moscow: Mir, 584.

8. Barabash, O. V., Dakhno, N. B., Shevchenko, H. V., Majsak, T. V. (2017). Dynamic models of decision support systems for controlling UAV by two-step variational-gradient method. 2017 IEEE 4th International Conference Actual Problems of Unmanned Aerial Vehicles Developments (APUAVD). doi: https://doi.org/10.1109/apuavd.2017.8308787

9. Chizhov, A. A. (2010). Sverhreleevskoe razreshenie. Vol. 2. Preodolenie faktora nekorrektnosti obratnoy zadachi rasseyaniya i proektsionnaya radiolokatsiya. Moscow: Krasand, 104.

10. Khudov, G. V. (2003). Features of optimization of two-alternative decisions by joint search and detection of objects. Problemy Upravleniya I Informatiki (Avtomatika), 5, 51-59.

11. Klimov, S. A. (2013). Metod povysheniya razreshayuschey sposobnosti radiolokatsionnyh sistem pri tsifrovoy obrabotke signalov. Zhurnal radioelektroniki, 1. Available at: http://jre.cplire.ru/jre/jan13/1/text.html

12. Lishchenko, V., Kalimulin, T., Khizhnyak, I., Khudov, H. (2018). The Method of the organization Coordinated Work for Air Surveillance in MIMO Radar. 2018 International Conference on Information and Telecommunication Technologies and Radio Electronics (UkrMiCo). doi: https://doi.org/10.1109/ukrmico43733.2018.9047560

13. Khudov, H., et. al. (2020). The Coherent Signals Processing Method in the Multiradar System of the Same Type Two-coordinate Surveillance Radars with Mechanical Azimuthal Rotation. International Journal of Emerging Trends in Engineering Research, 8 (6), 2624-2630. doi: https://doi.org/10.30534/ijeter/2020/66862020

14. Melvin, W. L., Scheer, J. A. (Eds.) (2012). Principles of Modern Radar: Advanced techniques. IET. doi: https://doi.org/10.1049/ sbra020e

15. Melvin, W. L., Scheer, J. A. (Eds.) (2013). Principles of Modern Radar: Volume 3: Radar Applications. IET, 820. doi: https:// doi.org/10.1049/sbra503e

16. Bezouwen, J., Brandfass, M. (2017). Technology Trends for Future Radar. Microwave Journal. Available at: http:// www.microwavejournal.com/articles/29367-technology-trends-for-future-radar

17. Thanh Huong, N. (2020). Beamforming Phased Array Antenna toward Indoor Positioning Applications. Advanced Radio Frequency Antennas for Modern Communication and Medical Systems. doi: https://doi.org/10.5772/intechopen.93133

18. Bhatta, A., Mishra, A. K. (2017). GSM-based commsense system to measure and estimate environmental changes. IEEE Aerospace and Electronic Systems Magazine, 32 (2), 54-67. doi: https://doi.org/10.1109/maes.2017.150272

19. Neyt, X., Raout, J., Kubica, M., Kubica, V., Roques, S., Acheroy, M., Verly, J. G. (2006). Feasibility of STAP for Passive GSM-Based Radar. 2006 IEEE Conference on Radar. doi: https://doi.org/10.1109/radar.2006.1631853

20. Willis, N. J. (2004). Bistatic Radar. IET. doi: https://doi.org/10.1049/sbra003e 
21. Khudov, H., Zvonko, A., Kovalevskyi, S., Lishchenko, V., Zots, F. (2018). Method for the detection of small-sized air objects by observational radars. Eastern-European Journal of Enterprise Technologies, 2 (9 (92)), 61-68. doi: https://doi.org/10.15587/ 1729-4061.2018.126509

22. Ruban, I., Khudov, H., Lishchenko, V., Pukhovyi, O., Popov, S., Kolos, R. et. al. (2020). Assessing the detection zones of radar stations with the additional use of radiation from external sources. Eastern-European Journal of Enterprise Technologies, 6 (9 (108)), 6-17. doi: https://doi.org/10.15587/1729-4061.2020.216118

23. Leshchenko, S. P., Kolesnyk, O. M., Hrytsaienko, S. A., Burkovskyi, S. I. (2017). Use of the ADS-B information in order to improve quality of the air space radar reconnaissance. Science and Technology of the Air Force of Ukraine, 3 (28), 69-75. doi: https:// doi.org/10.30748/nitps.2017.28.09

24. Saybel', A. G. (1958). Osnovy teorii tochnosti radiotekhnicheskih metodov mestoopredeleniya. Moscow: Oborongiz, 56.

25. Yeromina, N., Kravchenko, I., Kobzev, I., Volk, M., Borysenko, V., Lukyanova, V. et. al. (2021). The Definition of the Paramethers of Superconducting Film for Production of Protection Equipment Against Electromagnetic Environmental Effects. International Journal of Emerging Technology and Advanced Engineering, 11 (7), 38-47. doi: https://doi.org/10.46338/ijetae0721_06 